\title{
Formation of nanoscale ferromagnetic MnAs crystallites in low-temperature grown GaAs
}

\author{
P. J. Wellmann, ${ }^{\text {a) }}$ J. M. Garcia, J.-L. Feng, and P. M. Petroff \\ Materials Department, University of California in Santa Barbara, Santa Barbara, California 93106
}

(Received 5 May 1997; accepted for publication 2 September 1997)

\begin{abstract}
We report the formation of nanosize ferromagnetic MnAs crystallites imbedded in low-temperature grown GaAs using $\mathrm{Mn}^{+}$ion implantation and subsequent annealing. The structural and magnetic properties of the crystallites have been characterized by transmission electron microscopy, electron beam induced x-ray fluorescence, and superconducting quantum interference device magnetometry. After an optimized thermal annealing at $750{ }^{\circ} \mathrm{C}$, MnAs crystallites of $50 \mathrm{~nm}$ in size are formed. These nanomagnets show room temperature ferromagnetism. (c) 1997 American Institute of Physics. [S0003-6951(97)04343-X]
\end{abstract}

The formation of ferromagnetic nanosize particles $($ size $\approx 10-100 \mathrm{~nm})$ imbedded in a semiconductor host ${ }^{1,2}$ and the growth of so called diluted magnetic semiconductor layers, such as MnAsGa on GaAs, ${ }^{3,4}$ are of particular interest due to their application in hybrid magnetic semiconductor devices. Typical device applications are magnetic field sensors, switches, and memories (see for example Refs. 5 and 6). The underlying physical effects relevant to the hybrid structures with nanosize magnets are the Hall effect, the anisotropic magnetoresistive (AMR), and the giant magnetoresistive (GMR) effects. Recently, Shi et al. ${ }^{1}$ have shown the formation of $\mathrm{MnGa}$ ferromagnets imbedded in GaAs using $\mathrm{Mn}^{+}$ion implantation and subsequent heat treatment. These particles exhibit room temperature ferromagnetism. De Boeck et al. ${ }^{2}$ have reported the formation of MnAs crystallites in low-temperature (LT) GaAs introducing Mn during the molecular beam epitaxy MBE growth of the LT-GaAs layer. We have focused this study on the formation of MnAs crystallites in LT-GaAs using $\mathrm{Mn}^{+}$ion implantation and subsequent annealing.

We have grown a 200- or 400-nm-thick LT-GaAs layer (substrate temperature $250{ }^{\circ} \mathrm{C}$ ) on top of a $30 \mathrm{~nm} \mathrm{AlAs}$ layer and a $500 \mathrm{~nm} \mathrm{GaAs}$ buffer layer on a semi-insulating GaAs substrate. $\mathrm{Mn}^{+}$ions have been implanted at an energy of $E$ $=180 \mathrm{keV}$ and a dose ranging from $1 \cdot 10^{14}$ to 1 $\cdot 10^{16} \mathrm{~cm}^{-2}$. TRIM computer simulations give rise to a $\mathrm{Mn}^{+}$ ion distribution within the $200 \mathrm{~nm}$ LT-GaAs layer. The calculated penetration depth $R_{p}$ of the $\mathrm{Mn}^{+}$ions is $110 \mathrm{~nm}$, the distribution width $\Delta R_{p}$ is $50 \mathrm{~nm}$. Rapid thermal annealing has been performed at $600-900{ }^{\circ} \mathrm{C}(5-180 \mathrm{~s})$ under a forming gas atmosphere. The sample surface was covered with a $100 \mathrm{~nm}$ silicon nitride layer to prevent the evaporation of As during heat treatment. The structural properties have been investigated by transmission electron microscopy (TEM) and electron beam induced $x$-ray fluorescence (EDX). The magnetic properties have been characterized with a superconducting quantum interference device magnetometer (SQUID, $T=1.7-400 \mathrm{~K}, H=0- \pm 5 \mathrm{~T}$ ). To reduce the diamagnetic background signal of the GaAs substrate, we have lifted off the LT-GaAs:Mn layer by selectively etching away the AlAs layer with hydrofluoric acid. ${ }^{7}$

a)Electronic mail: wellmann@engineering.ucsb.edu
Figure 1(a) shows the cross sectional TEM image of a sample annealed at $750{ }^{\circ} \mathrm{C}(5 \mathrm{~s})$. The implanted Mn dose is $1 \cdot 10^{16} \mathrm{~cm}^{-2}$. The bright stripe $200 \mathrm{~nm}$ below the surface marks the $30 \mathrm{~nm} \mathrm{AlAs}$ which separates the $200 \mathrm{~nm} \mathrm{LT}$ -

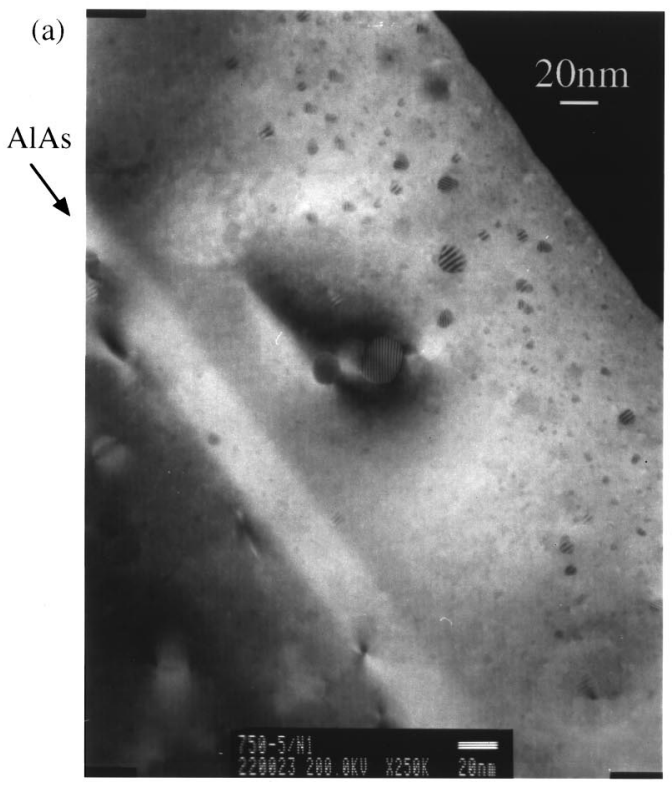

(b)

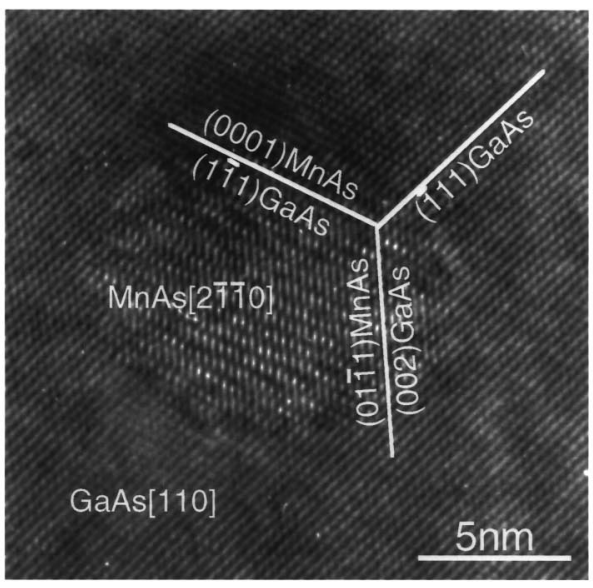

FIG. 1. (a) Cross sectional TEM image of LT-GaAs:Mn annealed at $750{ }^{\circ} \mathrm{C}$ (5 s) $\left([\mathrm{Mn}]=1 \cdot 10^{16} \mathrm{~cm}^{-2}\right)$. (b) High resolution TEM image of a single MnAs crystallite in LT-GaAs:Mn annealed at $750{ }^{\circ} \mathrm{C} \quad\left(\begin{array}{ll}5 & \text { s }\end{array}\right)$ $\left([\mathrm{Mn}]=1 \cdot 10^{16} \mathrm{~cm}^{-2}\right)$. 
TABLE I. Atomic fraction of $\mathrm{Ga}$, As, and $\mathrm{Mn}$ inside and outside a precipitate in LT-GaAs:Mn $\left([\mathrm{Mn}]=1 \cdot 10^{16} \mathrm{~cm}^{-2}\right)$ annealed at $750{ }^{\circ} \mathrm{C}(5 \mathrm{~s})$.

\begin{tabular}{|c|c|c|c|c|}
\hline & $\mathrm{Ga}[$ atom $\%]$ & As [atom $\%]$ & $\mathrm{Mn}[$ atom $\%]$ & Composition \\
\hline $\begin{array}{l}\text { LT-GaAs:Mn } \\
\text { (outside precipitate) }\end{array}$ & 52.6 & 46.7 & 0.7 & GaAs \\
\hline Precipitate & 5.2 & 49.9 & 44.9 & $\mathrm{GaAs}+\mathrm{MnAs}$ \\
\hline Precipitate $B$ & 25.7 & 46.3 & 27.9 & $\mathrm{GaAs}+\mathrm{MnAs}_{0.87} \mathrm{Ga}_{0.13}$ \\
\hline
\end{tabular}

GaAs:Mn from the GaAs buffer layer. After annealing we find the formation of nanosize particles. The average particle volume corresponds to a particle size of $10 \mathrm{~nm}$, the maximum size is up to $25 \mathrm{~nm}$. Figure 1(b) shows a high resolution TEM image of such a precipitate. The annealing process is very efficient in recrystallizing the amorphized LT-GaAs. The surrounding of the precipitates show few interface dislocations. The diffraction pattern of these particles indicate an epitaxial relationship with the GaAs matrix [Fig. 1(b)]: (0001)MnAs $\|(1 \overline{1} 1) \mathrm{GaAs}$ and $(01 \overline{1} 1) \mathrm{MnAs} \|(002) \mathrm{GaAs}$. Some smaller particles $($ size $=2-5 \mathrm{~nm})$ showed a different diffraction pattern; due to their small particle size it was not possible to estimate their epitaxial relationship with the GaAs matrix. However, larger MnAs crystallites ( size $>10 \mathrm{~nm}$ ) tend to induce the formation of dislocations due to a slightly lattice mismatch between the precipitate and the GaAs matrix. Therefore the dislocation density remains in the order of $10^{9} \mathrm{~cm}^{-2}$ after annealing. We have observed the formation of precipitates about $250 \mathrm{~nm}$ below the sample surface in the GaAs buffer layer. This layer has been grown at a substrate temperature of $600{ }^{\circ} \mathrm{C}$ and, unlike the LTGaAs layer grown at $250^{\circ} \mathrm{C}$, contains no excess As. Therefore we exclude the presence of As precipitates in the GaAs buffer layer. We suggest an outdiffusion of Mn towards the GaAs substrate and the formation of $\mathrm{Mn}$ rich precipitates during the annealing process at $750{ }^{\circ} \mathrm{C}(5 \mathrm{~s})$. No experimental data are available yet to support this hypothesis.

The chemical composition of the precipitates have been investigated by EDX measurements. We have used a small electron probe size (10 $\mathrm{nm}$ in diameter) to excite the $\mathrm{x}$-ray fluorescence. Table I summarizes the results for the precipitate shown in Fig. 1(b). Taking into consideration the surrounding LT-GaAs matrix, we find that this precipitate exhibits $\mathrm{Mn}$ and As with a ratio of $\mathrm{Mn}: \mathrm{As}=1: 1$, indicating that this particle is a MnAs crystallite. We also find that some precipitates contain a small amount of $\mathrm{Ga}$ (see precipitate B in Table I). Here the ratio of Mn:As is 1:0.6, indicating that precipitate $\mathrm{B}$ is a ferromagnetic phase of a $\mathrm{MnAs}_{n} \mathrm{Ga}_{m}$ alloy. Another possibility, the presence of an antiferromagnetic $\mathrm{Mn}_{2} \mathrm{As}$ phase, can be excluded from our magnetic measurements which show only ferromagnetic and superparamagnetic behavior.

We have carried out SQUID measurements of the magnetization $M(H)$ of LT-GaAs:Mn. Figure 2(a) shows the magnetization curves for various annealing conditions. The sample annealed at the lowest temperature $\left(600^{\circ} \mathrm{C}, 5 \mathrm{~s}\right)$ shows a small hysteresis loop. The magnetization does not saturate at high applied fields. This indicates that we have ferromagnetic particles in our LT-GaAs:Mn layer, but some particles may be too small in size $(<1-2 \mathrm{~nm})$ and give only rise to a superparamagnetic background signal. As we increase the annealing temperature the hysteresis loop increases too and becomes maximum for annealing at $750{ }^{\circ} \mathrm{C}$ $(5 \mathrm{~s})$. Now the magnetization saturates at high applied magnetic fields $(H>0.2 \mathrm{~T})$ indicating that almost all $\mathrm{Mn}^{+}$ions contribute to ferromagnetic crystallites. The Curie temperature $T_{c}$, which has been estimated from temperature dependent measurements of the remanence, is $T_{c}=330 \pm 5 \mathrm{~K}$. The value differs only slightly from bulk MnAs $\left[T_{c}(\mathrm{MnAs})=318 \mathrm{~K}\right]$. This confirms the result of the EDX measurement that the majority of the precipitates are MnAs crystallites.

As we increase the annealing temperature to $825{ }^{\circ} \mathrm{C}(5 \mathrm{~s})$ and $900{ }^{\circ} \mathrm{C}(5 \mathrm{~s})$ the saturation magnetization decreases [Fig. 2(a)]. One possible reason could be that a fraction of the $\mathrm{Mn}^{+}$ions diffuse during the heat treatment out of the LT-

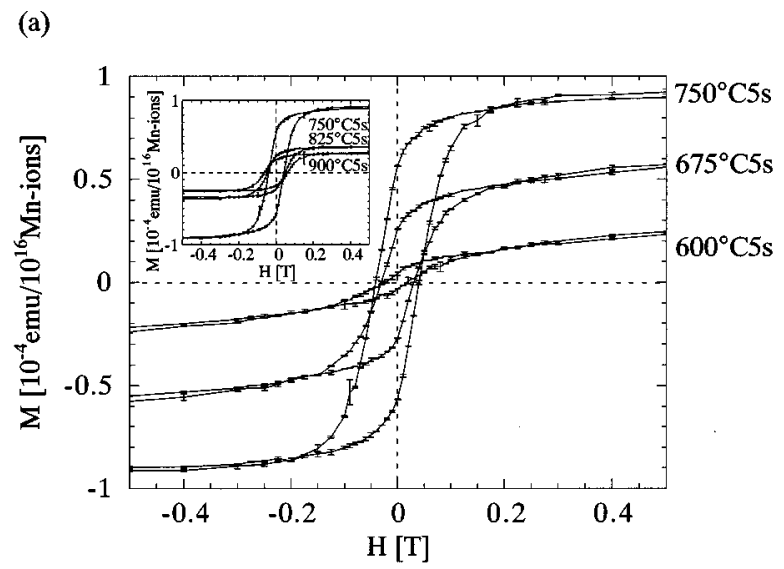

(b)

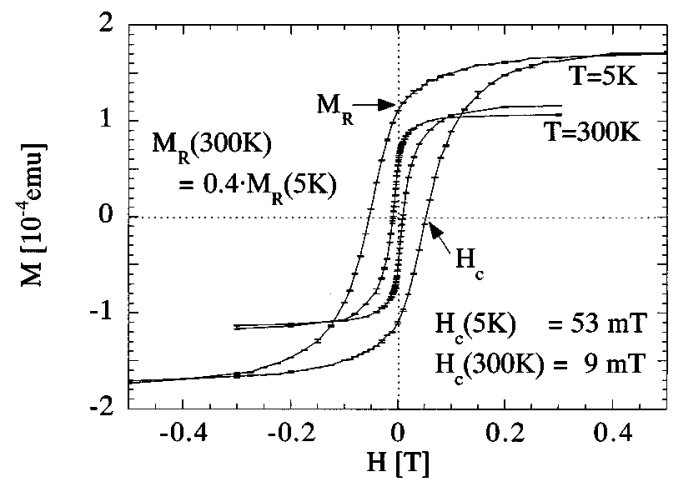

FIG. 2. (a) SQUID measurement at $T=5 \mathrm{~K}$ of the magnetization $M(H)$ of LT-GaAs:Mn $\left([\mathrm{Mn}]=1 \cdot 10^{16} \mathrm{~cm}^{-2}\right)$ for various annealing conditions $(T$ $\left.=600-900{ }^{\circ} \mathrm{C}, 5 \mathrm{~s}\right)$. The magnetization $M(H)$ of each sample has been normalized to $10^{16} \mathrm{Mn}$ ions. (b) SQUID measurement at 5 and $300 \mathrm{~K}$ of the magnetization $M(H)$ of LT-GaAs:Mn annealed at $750^{\circ} \mathrm{C}\left(\begin{array}{ll}180 & \text { s }\end{array}\right)$ $\left([\mathrm{Mn}]=1 \cdot 10^{16} \mathrm{~cm}^{-2}\right)$. 


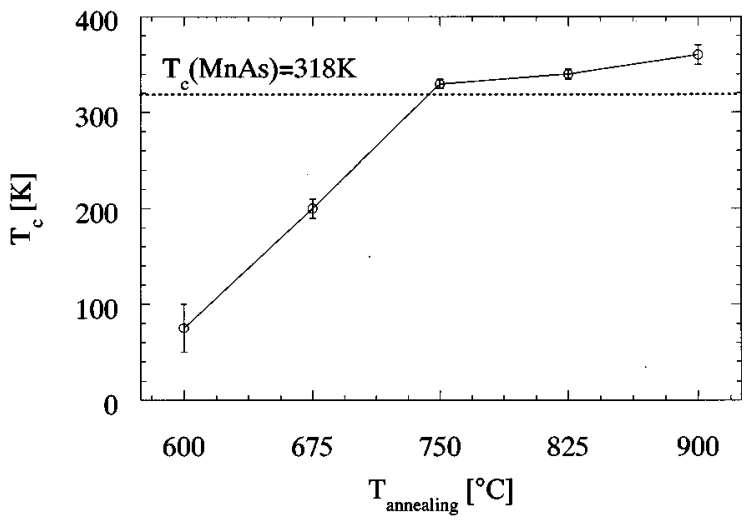

FIG. 3. Plot of the Curie temperature $T_{c}$ of LT-GaAs:Mn $\left([\mathrm{Mn}]=1 \cdot 10^{16} \mathrm{~cm}^{-2}\right)$ vs the annealing temperature $\left(T=600-900{ }^{\circ} \mathrm{C}, 5 \mathrm{~s}\right)$.

GaAs layer and into the GaAs buffer layer. We already mentioned the presence of some precipitates in the buffer layer when discussing the TEM image of the sample annealed at $750{ }^{\circ} \mathrm{C}(5 \mathrm{~s})$ [Fig. 1(a)]. In addition we should point out that there is evidence for a change of the chemical composition of the crystallites at higher annealing temperatures which may cause a decrease of the saturation magnetization too.

In Fig. 3 we have plotted the evolution of the Curie temperature versus the annealing temperature. The Curie temperature increases rapidly from $T_{c}\left(600{ }^{\circ} \mathrm{C}\right)=80 \mathrm{~K}$ to $T_{c}\left(750{ }^{\circ} \mathrm{C}\right)=330 \mathrm{~K}$. We attribute this to the growth of the precipitates and to the formation of the MnAs phase. Starting at an annealing temperature of $750{ }^{\circ} \mathrm{C}$ the Curie temperature increases further, but now slowly from $T_{c}\left(750{ }^{\circ} \mathrm{C}\right)=330 \mathrm{~K}$ to $T_{c}\left(900{ }^{\circ} \mathrm{C}\right)=360 \mathrm{~K}$. This behavior is attributed to a change of the chemical composition of the precipitates. The EDX analysis already showed that the sample annealed at $750{ }^{\circ} \mathrm{C}$ contains some precipitates with a small amount of Ga (precipitate $B$ in Table I). It is known from Shi et al. ${ }^{1}$ that the Curie temperature of $\mathrm{MnGa}$ precipitates in GaAs [annealed at $\left.920{ }^{\circ} \mathrm{C}(60 \mathrm{~s})\right]$ have a value above $400 \mathrm{~K}$. From this we conclude that in our experiment $\mathrm{MnAs}_{n} \mathrm{Ga}_{m}$ alloy precipitates may be formed at annealing temperatures higher than $750{ }^{\circ} \mathrm{C}$. The amount of $\mathrm{Ga}$ incorporated is supposed to increase with annealing temperature. The Curie temperatures of the $\mathrm{MnAs}_{n} \mathrm{Ga}_{m}$ particles are expected to be in between the values of $\operatorname{MnAs}\left(T_{c}=318 \mathrm{~K}\right)$ and $\operatorname{MnGa}\left(T_{c}>400 \mathrm{~K}\right)$.

For device applications of the MnAs nanosize ferromagnets imbedded in LT-GaAs, it is crucial to study the room temperature (RT) characteristics of the hysteresis loop.
Annealing at $750{ }^{\circ} \mathrm{C}$ for $5 \mathrm{~s}$ (without figure) yields a low room temperature coercivity $\left[H_{c}(5 \mathrm{~K})=41 \mathrm{mT} \rightarrow H_{c}(300 \mathrm{~K})\right.$ $=1 \mathrm{mT}]$ and remanence $\left[M_{R}(300 \mathrm{~K})=0.07 \cdot M_{R}(5 \mathrm{~K})\right]$. By increasing the annealing time from 5 to $180 \mathrm{~s}$ we were able to increase the MnAs particle size from 10 to $50 \mathrm{~nm}$ and therefore enhance the coercivity and remanence. Figure 2(b) shows the magnetization curves of a sample annealed at $750{ }^{\circ} \mathrm{C}$ for $180 \mathrm{~s}$. An increase of the room temperature coercivity by a factor of 9 is observed $\left[H_{c}(5 \mathrm{~s})\right.$ $\left.=1 \mathrm{mT} \rightarrow H_{c}(180 \mathrm{~s})=9 \mathrm{mT}\right]$. It is remarkable that the room temperature remanence $M_{R}$ remains $40 \%$ of the low temperature value. The conductivity of the LT-GaAs:Mn layer annealed at $750{ }^{\circ} \mathrm{C}(180 \mathrm{~s})$ is $110 \Omega^{-1} \mathrm{~cm}^{-1}$. The reported values of the coercivity, remanence, and conductivity make the MnAs nanosize crystallites imbedded in LT-GaAs possible candidates for hybrid ferromagnetic semiconductor devices if the diffusion of $\mathrm{Mn}$ is adequately controlled or used.

In conclusion we have reported the formation of nanosize ferromagnetic MnAs crystallites in LT-GaAs using $\mathrm{Mn}^{+}$ ion implantation and subsequent heat treatment. We have investigated the structural and the magnetic properties by TEM, EDX, and SQUID measurements. The best formation conditions of the MnAs crystallites in LT-GaAs:Mn are rapid thermal annealing at $750{ }^{\circ} \mathrm{C}$. The MnAs crystallites exhibit room temperature ferromagnetism. These nanomagnet characteristics could open the way to novel hybrid magnetic semiconductor structures.

We would like to thank D. D. Awschalom for fruitful discussions. This work has been supported by QUEST, an NSF Science and Technology center (Grant No. DMR91.20007). P.J.W. is a postdoctoral fellow of the Deutsche Forschungsgemeinschaft (DFG) J.M.G. is a postdoctoral fellow of the Spanish Ministry of Education and Science.

${ }^{1}$ J. Shi, J. M. Kikkawa, R. Proksch, T. Schaeffer, D. D. Awschalom, G. Medeiros-Ribeiro, and P. M. Petroff, Nature (London) 377, 707 (1995); J. Shi, J. M. Kikkawa, D. D. Awschalom, G. Medeiros-Ribeiro, P. M. Petroff, and K. Babcock, J. Appl. Phys. 79, 5296 (1996).

${ }^{2}$ J. De Boeck, R. Osterholt, A. Van Esch, H. Bender, C. Bruynseraede, C. Van Hoof, and G. Borghs, Appl. Phys. Lett. 68, 2744 (1996).

${ }^{3}$ H. Ohno, A. Shen, F. Matsakura, A. Oiwa, A. Endo, S. Katsumoto, and Y. Iye, Appl. Phys. Lett. 69, 363 (1996).

${ }^{4}$ T. Hayashi, M. Tanaka, T. Nishinaga, and H. Shimada, J. Appl. Phys. 81, 4865 (1997)

${ }^{5}$ J. De Boeck, T. Sands, J. P. Harbison, A. Scherer, H. Gilchrist, T. L. Cheeks, M. Tanaka, and V. G. Keramidas, Electron. Lett. 29, 421 (1993).

${ }^{6}$ J. M. Daughton, Thin Solid Films 216, 162 (1992).

${ }^{7}$ E. Yablonovitch, T. Gmitter, J. P. Harbison, and R. Bhat, Appl. Phys. Lett. 51, 2222 (1987). 